\title{
Scolia
}

Revue de linguistique

$30 \mid 2016$

Des connecteurs argumentatifs aux opérateurs discursifs

\section{La locution entre nous : un marqueur d'attitude polémique}

Adelaida Hermoso Mellado-Damas

\section{CpenEdition}

Journals

Édition électronique

URL : http://journals.openedition.org/scolia/521

DOI : 10.4000/scolia.521

ISSN : 2677-4224

Éditeur

Presses universitaires de Strasbourg

Édition imprimée

Date de publication : 19 juin 2016

Pagination : 139-154

ISBN : 978-2-86820-944-3

ISSN : 1253-9708

Référence électronique

Adelaida Hermoso Mellado-Damas, «La locution entre nous : un marqueur d'attitude polémique », Scolia [En ligne], 30 | 2016, mis en ligne le 09 avril 2019, consulté le 19 mai 2019. URL : http:// journals.openedition.org/scolia/521 ; DOI : 10.4000/scolia.521 


\title{
La locution entre nous: un marqueur d'attitude polémique
}

\author{
Adelaida Hermoso Mellado-Damas \\ Université de Séville \\ ahermoso@us.es
}

\section{Introduction}

Les auteurs spécialisés rangent le lexème entre nous dans la classe plus générale des adverbes d'énonciation du type sincèrement, franchement ou confidentiellement. Nølke (1990: 25), par exemple, inclut la locution entre nous dans la classe des "adverbiaux d'énonciation", plus concrètement dans le sous-groupe des "adverbiaux d'interlocuteurs ", lesquels «portent sur les protagonistes de l'acte illocutoire».

Marque-Pucheu, dans son article de (2010), fait intervenir la notion de "confidentialité» dans la description de cette locution; Molinier (2009: 13), pour sa part, range aussi entre nous à côté de confidentiellement et retient en plus un autre aspect de cette locution, à savoir celui du «rapport intersubjectif que le locuteur veut instituer avec l'interlocuteur (confidentialité, secret, connivence, etc.)».

De telles approches ne rendent pas compte cependant, à notre avis, de la vraie nature et de la spécificité fonctionnelle de cette expression. Elles ne permettent pas non plus de bien délimiter les domaines d'étude des différents types d'adverbes ou locutions d'énonciation, ceux-ci étant traités comme des sortes de synonymes regroupés dans une même catégorie tant grammaticale que sémantique et pragmatique. 
Du côté des dictionnaires, cette ambiguïté persiste: Le Petit Robert (2002: 875) donne comme paraphrase de entre nous «de vous à moi seulement, dans le secret», et traite la locution comme acception familière (par extension) de l'adverbe confidentiellement, posé comme son quasi-synonyme. Le Trésor de la Langue Française ${ }^{1}$ va dans le même sens et propose comme définition de entre nous «sans que cela ne soit connu d'autres personnes, sincèrement, franchement», ce qui, on le voit, suscite la même question: quelle est la spécificité sémantique et pragmatique de entre nous? En quoi diffère-t-il du reste des adverbes d'énonciation?

L'étude de notre corpus ${ }^{2}$ nous laisse voir que entre nous présente bien deux emplois, l'un comme constituant du SV, l'autre comme adverbe d'énonciation. La portée fonctionnelle semble donc en être claire; ce qui pose des problèmes, c'est la description des traits sémantico-pragmatiques de entre nous.

En m'appuyant sur un travail précédent (cf. Hermoso, à paraître) portant sur le contraste confidentiellement vs entre nous, je me propose ici de poursuivre l'analyse de entre nous afin d'en dégager d'autres traits sémantiques et pragmatiques susceptibles de lui être attachés.

Dans Hermoso (à par.) nous soutenons que, contrairement à la locution d'énonciation entre nous, l'adverbe confidentiellement ${ }^{3}$ n'exprime pas une attitude énonciative de la part du locuteurqui l'énonce: il porte sur les contenus des énoncés où il figure et non pas sur le sujet auteur desdits contenus. Dans le cas de l'adverbe, le locuteur se limite à adopter une attitude d'assertion, c'est-à-dire, à constater le caractère confidentiel des contenus énoncés.

Comme dans l'étude mentionnée ci-dessus, je me situe dans le cadre de la polyphonie esquissée dans Anscombre, Donaire et Haillet (2013). Suivant les postulats théoriques de la polyphonie, nous partons du principe qu'en structure profonde, tout énoncé se décompose en une série de points de vue $(p d v)$, chacun d'eux ayant un rôle à jouer

1 http://atilf.atilf.fr/dendien/scripts/tlfiv5/affart.exe?37;s=3643180920;?b=0, $12 / 06 / 2015$.

2 Notre corpus compte 250 occurrences environ extraites d'œuvres littéraires françaises postérieures à 1940, de textes de presse (Le Monde Diplomatique), ou recueillies sur internet.

3 Ainsi que d'autres unités comme concrètement et objectivement. 
dans la stratégie discursive mise en place par le locuteur dudit énoncé. Il s'agira de voir dans quelle mesure la présence de entre nous dans un énoncé particulier détermine l'agencement des différents points de vue convoqués lors de son énonciation.

Dans une première partie, j'appliquerai une série de critères formels qui mettront en évidence le caractère extra-prédicatif et thématique de la locution entre nous ${ }^{4}$, la distinguant du syntagme prépositionnel interne au SV. Dans une deuxième partie, en m'appuyant sur certaines propriétés sémantiques et pragmatiques, je partirai de l'hypothèse que la locution entre nous ouvre un espace polémique où deux $p d v$ s'opposent, et décide du statut de la marque de deuxième personne contenue dans l'énoncé qu'elle accompagne. Entre nous évoque ainsi une attitude énonciative que les interlocuteurs manifestent à l'égard des $p d v$ mis en place.

\section{Caractéristiques distributionnelles de entre nous}

Comme on l'a déjà fait remarquer dans l'introduction, d'après la bibliographie consultée et l'étude du corpus, entre nous semble présenter deux emplois bien différenciés:

Ea) Comme constituant du syntagme verbal:

1) Il n'y avait ni hostilité ni amitiéentre nous. (Osmont, Éléments incontrôlés, 2012)

Eb)Comme adverbe d'énonciation, à l'instar de franchement et sincèrement:

2) Entre nous, confidentiellement, je pense qu'il y aura une autopsie... par précaution, vous comprenez? (Simenon, Les vacances de Maigret, 1948)

Voyons en quoi diffèrent ces deux emplois.

En tant que constituant du SV, entre nous peut être extrait dans une structure clivée (3a) et se trouve sous la portée de la négation ( $3 b$ ) et de l'interrogation $(3 \mathrm{c})$ :

4 Je traiterai ici les locutions soit dit entre nous, entre nous soit dit et entre nous comme des synonymes et laisserai pour une autre occasion la question de l'évolution d'une expression vers l'autre et du phénomène de grammaticalisation, selon lequel, comme le signale Pop (2001:17), «une proposition sémantiquement pleine se réduit à une formule». 
3) Il y a trop de distance entre nous.

a. C'est entre nous qu'il y a trop de distance (et non pas entre vous).

b. Il n'y a pas trop de distance entre nous (mais entre vous).

c. Est-ce qu'il a trop de distance entre nous (ou entre vous)?

Mais la caractéristique distributionnelle la plus saillante de ce syntagme prépositionnel, comme le suggère Marque-Pucheu (2010), est que le pronom personnel nous est susceptible de commuter avec d'autres pronoms, c'est-à-dire qu'il entre dans un paradigme pronominal:

4) Il y a trop de distance entre nous [vous/eux/elles].

Enfin, une autre caractéristique du segment entre nous est qu'il peut être déplacé en tête de phrase sans que cela altère son statut interne au prédicat $^{5}$ :

5) Entre nous, il y a trop de distance.

6) Entre nous, il ne peut y avoir de relation de confiance.

En tant qu'adverbe d'énonciation, la locution entre nous n'accepte pas d'être le focus d'une phrase clivée (7a), ni d'être sous la portée de la négation (7b) ou de l'interrogation (7c); enfin, elle ne peut pas non plus occuper une position après le verbe (ou le COD, si c'est le cas) (7d):

7) Entre nous, je pense qu'il y aura une autopsie.

a. ${ }^{*}$ 'est entre nous que je pense qu'il y aura une autopsie.

b. ${ }^{\star} I l$ n'y aura pas une autopsie entre nous.

c. ${ }^{\star}$ Est-ce qu'il y aura une autopsie entre nous?

d. ${ }^{\star}$ Je pense qu'il y aura une autopsie entre nous.

En ce qui concerne le critère de la commutation, entre nous d'énonciation n'entre pas dans un paradigme pronominal:

e. Entre nous [ ${ }^{*} e u x /{ }^{*}$ vous / ${ }^{*}$ elle], je pense qu'il y aura une autopsie.

Pour en finir avec les caractéristiques distributionnelles de ces deux emplois de entre nous, selon Marque-Pucheu (2010: 4), dans le cas de la locution énonciative, «nous ne pouvons pas recourir à une construction détachée où les pronoms sont coordonnés ( ${ }^{\star}$ Entre nous, toi et moi, j'en doute)»; contrairement au cas de entre nous constituant de SV, "où la coordination introduit une symétrie que

5 Sauf bien évidemment lorsqu'il accompagne le verbe être, auquel cas il devient un constituant obligatoire, face aux autres cas (exemples (5) et (6) où il est facultatif: «On est entre nous» / « ${ }^{\star}$ Entre nous on est»). 
corrobore la permutation (Moi et toil Toi et moi, nous sommes entre nous)». Comme le signale l'auteur, si l'on veut insister sur l'adverbe d'énonciation, on est obligé de recourir à la formule «de toi à moi»: «entre nous, de toi à moi, je pense qu'il y aura une autopsie». Or, il faut préciser que la fonction syntaxique du pronom y joue un rôle décisif: si la commutation est possible dans "Toi et moi, nous sommes entre nous", c'est parce que les pronoms coordonnés reprennent le nous sujet de la phrase. Lorsque nous ne remplit pas la fonction sujet, cette commutation se révèle impossible: " [ ${ }^{*}$ Toi et moi], il y a trop de distance entre nous, [entre toi et $m o i{ }^{\star}{ }^{\star}$ toi et $\left.m o i\right] »$; autrement dit, la répétition de entre nous constituant en apposition exige aussi la présence de la préposition correspondante.

Pour résumer, on dira que, dans ces deux emplois, Ea) et Eb), le segment entre nous pourrait être paraphrasé par:

Ea) Il y trop de distance entre toi et moi ${ }^{6}$.

$\mathrm{Eb})$ Que cela reste entre nous / Je le dis entre nous, je pense qu'il y aura une autopsie.

\section{Caractéristiques sémantico-pragmatiques de entre nous}

Deux caractéristiques, à la fois sémantiques et pragmatiques, sont attachées à entre nous: l'une concernant le type d'attitude énonciative que le sujet parlant manifeste envers les contenus énoncés; l'autre relative au rôle joué par l'interlocuteur dans la stratégie discursive mise en place à travers son emploi.

\subsection{Entre nous et l'énonciation: assertion vs monstration}

Selon l'approche polyphonique que nous adoptons ${ }^{7}$, tout énoncé se décompose en une série de points de vue, notés $p d v$, chaque $p d v$ ayant une source $[\mathrm{x}]$, et un contenu $\{\mathrm{p}\}$. Une description sémantique devrait ainsi rendre compte du nombre et de la nature des $p d v$ sousjacents à un énoncé donné ainsi que de la relation que le locuteur dudit

6 Remarquons que l'emploi de entre toi et moi à la place de l'expression entre nous ne serait pas pour nous une paraphrase à proprement parler mais plutôt une variante pronominale.

7 Cf. Anscombre, Donaire et Haillet (2013). 
énoncé entretient avec ces $p d v$. Pour donner un exemple simplifié, dans l'énoncé "À mon avis, Paul est un bon chercheur», la locution à mon avis convoque un $p d v$ dont le contenu $\{\mathrm{p}\}$ serait «Paul est un bon chercheur» et la source $[\mathrm{x}]$ le locuteur de l'intégralité de l'énoncé. Dans ce cas, la relation existant entre la source et le contenu est une relation d'adhésion, mais on peut en trouver d'autres : assertion, monstration, etc.

C'est la prise en compte de cette relation qui nous semble essentielle à l'étude de l'unité ici concernée, en particulier la différence existant entre l'attitude d'assertion et l'attitude de monstration.

Il y a des unités de la langue qui sont particulièrement aptes à signaler une attitude de monstration lorsqu'elles apparaissent en position détachée en tête de phrase. C'est le cas des adverbes franchement et sincèrement. Par exemple, la différence entre a) Je te dis sincèrement que j'en suis déçu et b) Sincèrement, j'en suis déçu, réside dans la manière dont est présentée l'attitude sincère du locuteur: dans a) elle est décrite, dans b), en revanche, elle est montrée ${ }^{8}$.

Comme l'affirme Anscombre (1992: 45), à cette fonction de marqueur de d'attitude énonciative de monstration viendrait s'ajouter une fonction de marqueur "d'espace discursif»; sincèrement et franchement constituent alors «le cadre dans lequel s'inscrit ce qui suit». En tant que cadre thématique, les adverbes sincèrement et franchement présentent, entre autres, deux caractéristiques distributionnelles ${ }^{9}$ : $\mathrm{Ca}$ ) ils ne peuvent être enchâssés dans une complétive dont le verbe régissant n'est pas un verbe de dire; $\mathrm{Cb}$ ) ils ne peuvent pas être ce sur quoi on enchaîne. Voyons à travers des exemples quels sont les résultats de l'application de ces deux critères.

Ca) Enchâssement:

8) Sincèrement $[+$ Franchement $]$, je suis étonné que Paul ait parlé contre Marie.

a. Je suis étonné que Paul ait parlé sincèrement [+ franchement] contre Marie.

8 C'est aussi bien la différence qui s'établit entre dire a) «je trouve ce livre magnifique» et s'exclamer b) «C'est un livre magnifique!»; dans les deux cas il est question du caractère magnifique du livre, la différence réside en ce que dans a) ce caractère est affirmé, décrit par le locuteur, tandis qu'en b) il est montré (cf. Anscombre, 2013).

9 On ne retient ici que les deux caractéristiques qui nous semblent être les plus représentatives de ce type d'emploi adverbial, mais ce ne sont pas les seules. Pour une étude plus en détail de cette question, voir Anscombre (2001: 145). 
b. Je suis étonné que, ${ }^{*}$ sincèrement $\left[+{ }^{\star}\right.$ franchement $]$, Paul ait parlé contre Marie.

Cb) Enchaînement ${ }^{10}$ :

9) Je suis sincère $[+$ franc] en te disant que ta cravate te va mal, [tu devrais en changer/parce que c'est pour ton bien].

10) Sincèrement [+ franchement], ta cravate te va mal, [tu devrais en changer $/{ }^{*}$ parce que c'est pour ton bien].

Examinons maintenant le comportement de entre nous par rapport à ces deux critères distributionnels :

Cb) Enchaînement:

11) a. Il y a trop de distance entre nous [et non pas entre eux/ et non pas trop de chagrin].

b. Entre nous il y a trop de distance [et non pas entre eux/et non pas trop de chagrin].

c. Entre nous, ce livre ne vaut rien, [ne l'achète pas $/{ }^{*}$ par discrétion].

d. Que cela reste entre nous, ce livre ne vaut rien, [ne l'achète pas $/{ }^{*}$ par discrétion].

La série (11) montre que dans (a) et (b), la rectification peut enchaîner aussi bien sur le syntagme prépositionnel entre nous que sur le syntagme nominal trop de distance. Lorsque entre nous est adverbe d'énonciation, en revanche, il ne peut pas être ce sur quoi on enchaîne, ni comme locution en position frontale (c), ni comme constituant d'une phrase d'énonciation explicite (d). Pour ce qui est de sa position en incise médiane, seule la phrase d'énonciation paraît accepter l'enchaînement (exemple 13), tandis que la locution, elle, la refuse (exemple 12):

12) Ce livre est nul (ne vaut rien), entre nous, [ne l'achète pas /??par discrétion].

13) Ce livre est nul (ne vaut rien), que cela reste entre nous, [ne l'achète pas / par discrétion].

Ca) Enchâssement:

14) a. Je suis étonné qu'il y ait cette distance entre nous.

b. Je suis étonné qu'entre nous il y ait cette distance.

c. Entre nous, je suis étonné que Paul ait parlé contre Marie.

10 Les exemples (9) et (10) sont empruntés à Anscombre (2001: 147). 
d. Je suis étonné que, entre nous, Paul ait parlé contre Marie.

En ce qui concerne le critère de l'enchâssement, la série (14) montre bien que la locution ne peut être enchâssée dans une complétive, face au syntagme prépositionnel interne au prédicat.

Les résultats des critères appliqués prouvent qu'il s'agit dans ce cas d'un adverbe d'énonciation au même titre que franchement et sincèrement, c'est-à-dire, qu'on doit considérer entre nous en tant qu'expression d'une attitude de monstration.

Mais il y a un autre trait sémantico-pragmatique qui rapproche entre nous de sincèrement et franchement: une attitude de monstration passe par l'implication directe de l'instance de première personne dans l'opération discursive mise en jeu. Ce qui n'est pas forcément le cas pour des unités discursives qui impliquent une description ou une assertion.

Si l'on revient sur notre exemple (2), repris ci-dessous, on observe que la locution entre nous montre l'attitude du locuteur, alors que l'adverbe confidentiellement la décrit ${ }^{11}$. La locution convoque un point de vue préalable auquel s'oppose un deuxième point de vue selon lequel "il y aura une autopsie». L'expression entre nous ouvre ainsi un espace polémique.

2) (Le commissaire Maigret au médecin): - Entre nous, confidentiellement, je pense qu'il y aura une autopsie.

Remarquons que lors du passage du discours direct au discours indirect (exemple 15), seul l'adverbe peut être rapporté tandis que la locution doit être soit supprimée, soit remplacée par une glose approximative, par exemple «contrairement à ce que l'on pourrait en penser/croire» :

15) Le commissaire Maigret dit [ ${ }^{*}$ entre nous/confidentiellement] au médecin que, [contrairement à ce que l'on pourrait croire], il pense qu'il y aura une autopsie...

11 Ce qui nous conduit à affirmer qu'un seul et même énoncé peut être accompagné de deux marqueurs discursifs présentant chacun une attitude énonciative différente, quoique susceptible d'être adoptée par le même locuteur. Or, toutes les attitudes ne sont pas cumulables. L'attitude d'adhésion signalée ci-dessus est incompatible avec

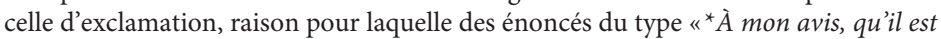
beau, cet homme!» ou « ${ }^{\star}$ 'après moi, comme tu es belle!» sont inacceptables. 
Autrement dit, on ne peut pas rapporter une expression qui représente une attitude de monstration, l'instance de la première personne se perd et, par conséquent, la relation entre source et contenu change complètement ${ }^{12}$.

Une fois établi le statut d'adverbe de monstration de la locution entre nous, voyons en quoi consiste exactement l'attitude énonciative qu'il évoque.

\subsection{Entre nous: un marqueur d'attitude énonciative}

L'analyse en détail du corpus nous montre que, dans le co-texte droit de entre nous (ou équivalents), il y a toujours des traces de l'engagement subjectif du locuteur dans son discours, comme celles soulignées dans les séquences (16) et (17):

16) Aussi, je tiens à commencer par vous rassurer de suite, Polux va pour le mieux et se porte très bien. Soit dit entre nous, vous auriez pu trouver un nom un peu moins con pour un castor, enfin... personne n'est parfait. (Fatrov, Dernier avertissement avant saisie! 2013)

17) J'avais demandé à Zan de venir dîner ce soir mais elle a rendezvous avec son ex-mari, ce qui est une erreur entre nous soit dit. Il l'accuse d'avoir laissé son fils. (Higgins Clark, Quand reviendrastu? 2011)

En effet, comme le montrent ces exemples, le locuteur émet un jugement de valeur qui se traduit par une évaluation subjective d'un fait ou d'un événement. Dans (16) l'évaluation porte sur le fait d'appeler un castor "Polux»; dans (17) c'est le fait que Zan fixe un rendez-vous avec son ex-mari qui se voit critiqué. On constate aussi que, dans la plupart des occurrences de entre nous, le jugement émis par le locuteur implique une dévaluation: le verbe douter (exemple 18), le substantif erreur (exemple 17) ou les adjectifs con (exemple 16), chiant (exemple 19), singulière (exemple 24), entre autres, apparaissent en cooccurrence avec la locution dans le corpus. L'énoncé (20) ci-dessus est aussi un exemple de cette particularité de entre nous:

12 Remarquons que cette transposition s'avère aussi impossible avec la phrase d'énonciation correspondant à entre nous: "Le commissaire Maigret dit [ ${ }^{*}$ que cela reste entre nous] au médecin qu'il pense qu'il y aura une autopsie». 
18) Mais, entre nous soit dit, Ronny, si Faye était encore vivante, je doute fort qu'elle apprécierait tes manœuvres. (Bevarly, Fiancée malgré elle, 2009).

19) Au conseil, tous les collègues auront envie de se défouler, ça ne va pas faire un pli. Soit dit entre nous, c'est vrai qu'il est chiant. (Jonquet, Ils sont votre épouvante et vous êtes leur crainte, 2006)

20) Je te crois. Toute façon, entre nous soit dit, je ne peux pas le sentir, ce clébard. Toujours à venir me renifler avec sa truffe baveuse. Il m'en met plein partout sur le costume. (Osmont, Éléments incontrôlés, 2012)

En m'appuyant sur ces données, je fais l'hypothèse que entre nous ouvre un espace polémique où s'opposent deux points de vue de contenus contraires. Observons à cet égard les exemples fabriqués (21) et $(22)$ :

21) On dit que Paul est un bon chercheur. Entre nous, il est plutôt médiocre.

22) On dit que Paul est un bon chercheur. ?Entre nous, il est très bon.

Pour que l'enchaînement dans (22) soit senti comme naturel, on doit imaginer un contexte, un peu forcé, où l'opinion selon laquelle "Paul est un bon chercheur» serait en grande minorité par rapport à "Paul est un chercheur médiocre».

Ajoutons à cette explication que l'expression on dit que, dans une structure du type [On dit que $\mathrm{X}$ ], n'implique pas forcément le locuteur comme faisant partie de la source énonciative de $\mathrm{X}^{13}$, ce qui autorise sa combinaison avec entre nous dans (21). En effet, la combinaison de la locution et d'un énoncé qui exprime un accord avec ce qui précède produit un résultat douteux - ce qui s'explique par le fait que l'emploi de entre nous met en place un espace discursif polémique.

Les deux exemples (23) et (24) tirés du corpusillustrent très bien cette particularité sémantico-pragmatique de entre nous:

23) Ben, la conversion des yens vers l'euro! S'indigne-t-elle. Ça prend du temps, et entre nous, des budgets européens en yen, ça n'a pas beaucoup de sens. (Shepard, Absolument débordé, 2010)

24) Pendant que je vous envoie ces lignes, la Princesse répondait à Mallefille dont la lettre m'a paru (entre nous soit dit) bien sin-

13 Cette source étant représentée par un ON-locuteur (cf. Anscombre, 2005). 
gulière, pour ne pas dire d'avantage. (Marix-Spire, Les romantiques et la musique: le cas George Sand, 1804-1830, vol. 1, 1953)

Dans (23), la locution introduit un $p d v$ qui s'oppose à un point de vue attribué à d'autres selon lequel des budgets européens en yen, ça aurait du sens. Dans l'exemple (24), le point de vue sous-jacent et dont la glose serait quelque chose comme "Mallefille a écrit une lettre correcte (à la Princesse)» se trouve contesté et critiqué par le locuteur du tout. Là encore, entre nous occupe un contexte dépréciatif ${ }^{14}$.

Ce trait pragmatique rapproche l'expression entre nous de l'adverbe d'énonciation franchement, lequel ouvre aussi un espace polémique. Tel que suggéré par Donaire (2006), l'adverbe franchement présente un espace discursif polémique, c'est-à-dire qu'il convoque un point de vue préalable, auquel s'opposent les contenus qu'il accompagne. C'est la raison de la différence existant entre (25) et $(26)^{15}$ :

25) A: - J'ai fait un gros effort. B : - ?Franchement, je te crois.

26) A: - J'ai fait un gros effort. B: - Franchement, je ne te crois pas.

Le problème avec la séquence (25) consiste en ce que le locuteur exprime une attitude de monstration qui traduit une relation d'opposition entre un $p d v_{1}$ attribué à d'autres, dont les contenus seraient " $A$ a fait un grand effort » et un $p d v_{2}$ sous-jacent à l'intervention de $\mathrm{B}$, selon lequel «A n'a pas fait un grand effort»; franchement agit ici en tant que cadre polémique où est inséré le contenu «je te crois», ce qui pose problème.

Curieusement, l'on constate que la substitution de l'adverbe franchement à la locution entre nous dans les exemples (23) et (24) commentés ci-dessus n'entraîne pas de changement de sens significatif ${ }^{16}$. La présence de franchement convient parfaitement:

14 Précisons cependant que entre nous n'occupe pas toujours un contexte dépréciatif. Il $\mathrm{y}$ a des cas où la locution limite son apport à présenter un point de vue comme étant contraire à une opinion censée être majoritaire ou bien partagée de toute une communauté. Dans ces cas, le locuteur exprime son opinion - minoritaire - par un prédicat modal du type je crois/ je pense. Voici un exemple: «Entre nous soit dit, je crois que Bartley avait le béguin pour elle. Il lui avait dit qu'elle était plus jolie que la plupart des filles qu'elle maquillait». (Higgins Clark, Quand reviendras-tu?, 2011). Cf. aussi l'exemple 2), $\$ 1$.

15 Ces exemples sont empruntés à Donaire (2006).

16 Notons que cette même commutation vaut pour les exemples (16), (17) et (18) (\$2.2). 
23) a. - Ben, la conversion des yens vers l'euro! S'indigne-t-elle. Ça prend du temps, et entre nous [+ franchement], des budgets européens en yen, ça nia pas beaucoup de sens.

24) a. Pendant que je vous envoie ces lignes, la Princesse répondait à Mallefille dont la lettre mia paru (entre nous soit dit $[+$ franchement $]$ ) bien singulière.

Mais ce trait d'opposition n'est pas le seul qui nous aidera à décrire la spécificité sémantico-pragmatique de entre nous. Il y a une autre propriété qui caractérise la locution et qui la différencie de franchement: il s'agit du rôle qu'elle assigne à l'interlocuteur du message véhiculé par l'énoncé.

\subsection{Entre nous en tant que marqueur interlocutif}

Si franchement et entre nous partagent le paramètre concernant la relation entre la source et le contenu, c'est-à-dire l'attitude adoptée de la part du locuteur qui les énonce, ils diffèrent quant à l'identification d'une telle source. Franchement, on l'a dit, introduit un $p d v$ attribué à d'autres, c'est-à-dire un $p d v$ dont la source n'est pas le locuteur; entre nous, pour sa part, introduit aussi un $p d v$ attribuée à une source distincte du locuteur mais dont l'interlocuteur se trouverait aussi exclu. Dans l'exemple (27) la commutation de franchement et entre nous s'avère impossible; le cadre instauré dans ce cas concerne uniquement le locuteur (B):

27) A- Voulez-vous une bière?

B- [Franchement $+{ }^{*}$ entre nous], merci, mais jai du travail.

Pour donner une explication à cette apparente contradiction, je ferai ici recours à la distinction établie chez Ducrot (1980: 29-38) entre l'auditeur du discours, appartenant au niveau de la réalité empirique, et le destinataire en tant que personnage de discours auquel l'énonciateur adresse son message et que l'énoncé pose comme relié à son apparition ${ }^{17}$.

Si l'on observe à l'égard de cette distinction l'exemple $(28)^{18}$,

28) Entre nous, tu as eu tort de refuser.

17 Rappelons que, pour Ducrot (1980: 38), l'énonciateur serait «la personne à qui est attribuée la responsabilité d'un acte illocutoire» et le destinataire «celle à qui cet acte est censé s'adresser ".

18 Exemple emprunté à Nølke (1989: 50). 
l'on constate que l'instance de deuxième personne tu agglutine deux entités différentes que l'on pourrait dissocier : l'interlocuteur - auditeur chez Ducrot - de l'énoncé, lequel pourrait être désigné par le pronom te d'une phrase d'énonciation explicite du type "je te dis que tu as eu tort de refuser» et le destinataire de l'acte accompli lors de son apparition, qui serait désigné par le pronom tu de la phrase sousjacente "tu as refusé». Cette ambiguité référentielle autorise la présence de la locution entre nous, laquelle déconsidère le tu-destinataire, sujet $\mathrm{du}$ verbe refuser, et retient uniquement le tu-interlocuteur, auteur du verbe avoir tort. Ces faits nous emmènent à conclure que entre nous porte sur le contenu posé et non pas sur les contenus présupposés de l'énoncé qui l'accueille.

Lorsqu'un tel amalgame pronominal n'existe pas, la présence de entre nous est impossible. Si l'on observe sous cet angle l'exemple (27), commenté ci-dessus, on comprend tout de suite pourquoi il refuse la présence de entre nous: le vocatif merci de (27) recevrait une paraphrase du type "je vous remercie», laquelle ne supporte pas la présence de la locution ( "Entre nous, je vous remercie»); l'instance de deuxième personne (avec marque de politesse) vous réfère ici uniquement au destinataire du message proféré (de l'acte de remerciement), ou ce qui est le même, dans (27) le destinataire en tant que personnage de discours coïncide avec l'interlocuteur en tant qu'être du monde sans qu'une séparation entre ces deux entités ne puisse être opérée, raison pour laquelle la présence de entre nous ne convient pas.

Observons sous ce même angle les exemples suivants:

29) ??Entre nous, tu as refusé19.

a. ??Je te dis que tu as refusé.

30) Entre nous, c'est toi qui as refusé.

a. Je te dis que c'est toi qui as refusé.

Là encore on assiste au même phénomène: dans (29) il n'est pas possible d'interpréter le pronom tu comme référant à l'interlocuteur du message communiqué, la phrase d'énonciation explicite (29a) est inacceptable (ou dépourvue de sens); l'exemple

19 Soulignons que c'est la présence du pronom de deuxième personne $t u$ qui rend cette séquence maladroite; si on le remplace par un pronom à la troisième personne, la phrase devient grammaticale: "Entre nous, il/elle a refusé», ainsi que la phrase d'énonciation correspondante "Je te dis qu'il/elle a refusé». 
(30), en revanche, semble beaucoup plus naturel, la dissociation entre interlocuteur / destinataire du message est tout à fait viable: dans ce cas, l'interlocuteur est représenté par le pronom tonique «toi», tandis que le destinataire est désigné par le pronom relatif sujet «qui». Quant à la phrase d'énonciation (30a), elle contient deux pronoms personnels qui présentent une coréférence: le $\mathrm{COI}$ «te» et la forme tonique «toi». Comme dans l'exemple (28), on constate ici que entre nous ne porte pas sur les contenus présupposés mais sur les contenus posés de l'énoncé qui l'accueille.

Si l'on observe la séquence (31), laquelle contient le marqueur soit dit en passant:

31) [...] que notre chère camarade Ridot a bien voulu se charger de préparer pour nous lors de notre dernière réunion, à laquelle tu n'assistais pas, soit dit en passant [ ${ }^{*}$ soit dit entre nous]. (Chabrol, La Folie des miens, 1977)

on s'aperçoit que la substitution de soit dit entre nous à soit dit en passant s'avère impossible: l'instance de deuxième personne soulignée «tu» ne réfère pas ici à l'interlocuteur du message, mais au destinataire de celui-ci, i.e. à la personne à laquelle est adressé l'acte illocutoire de reproche émis par l'auteur de (31).

Ajoutons une dernière remarque sur entre nousqui va dans le même sens. Comme le montrent les exemples (32), (32a), (33) et (33a), la présence de comme tu sais est incompatible avec celle de entre nous:

32) Entre nous, ta femme te trompe.

a. ${ }^{\star}$ Entre nous, comme tu sais, ta femme te trompe.

33) Entre nous, j'ai gagné à la loterie.

a. ${ }^{\star}$ Entre nous, comme tu sais, j'ai gagné à la loterie.

Comme tu sais convoque un $p d v$ qui traduit un savoir partagé des interlocuteurs et place ainsi l'instance de deuxième personne comme étant co-responsable et donc destinataire du message communiqué.

L'auteur de [Entre nous, X] s'adresse à l'interlocuteur de $\mathrm{X}$ et non pas au destinataire du message communiqué dans $\mathrm{X}$. La locution entre nous décide du statut discursif de la marque de deuxième personne contenue dans l'énoncé qu'il accompagne.

Ce que nous venons de préciser à propos de entre nous nous conduit à nous opposer à Marque-Pucheu (2010: 11) lorsqu'elle affirme que entre nous «invite l'allocutaire/énonciataire à écouter un point de 
vue confidentiel et, le cas échéant, à exprimer lui-même un avis/une information sous le sceau dela confidence». Pour nous, il n'en est rien: d'un côté, le locuteur de entre nous ne s'adresse pas à l'allocutaire / énonciataire du message communiqué, mais à l'interlocuteur présent dans la situation d'énonciation; d'un autre côté, l'emploi de entre nous ne concerne pas la notion de confidence; on peut très bien imaginer une séquence du type Paul est un bon chercheur. Mais, entre nous, on dit qu'il est un peu brouillon, où l'expression on dit que attribue les contenus «Paul est un peu brouillon» à un ON-locuteur, auquel le locuteur peut ou non s'identifier mais qui, en tout cas, n'est en rien confidentielle. On assiste là à une espèce de transfert plus ou moins intuitif et direct du sémantisme propre à l'adverbe confidentiellement à la description théorique de la locution, sans qu'aucun critère spécifique ne vienne étayer l'hypothèse.

\section{Conclusion}

Notre analyse a montré que entre nous est un marqueur discursif qui implique une attitude de monstration de la part du locuteur envers les contenus proférés. Cette attitude énonciative, en tant que cadre discursif, ouvre un espace polémique où placer un énoncé donné. Selon notre approche polyphonique, la locution entre nous convoque deux points de vue qui s'opposent: un premier $p d v$ dont la source exclut les interlocuteurs et un second $p d v$ dont la source implique directement le locuteur. Entre nous présente une autre particularité, on l'a vu, qui revient à avoir la capacité de distribuer les différents rôles discursifs lors de la production de l'énoncé qui l'accueille. On a pu constater aussi que l'application de critères sémantico-pragmatiques pertinents s'avère nécessaire pour réussir une description complète et exhaustive de cette unité de la langue.

\section{Bibliographie}

ANSCOMBRE J.-C. (1992), Imparfait et passé composé: des forts en thème/propos, L'information grammaticale 55, 43-53.

ANSCOMBRE J.-C. (2001), À coup sûr/Bien sûr: des différentes manières d'être sûr de quelque chose, Recherches en Linguistique et Psychologie cognitive 16, 135-160. 
ANSCOMBRE J.-C. (2005), Le ON-locuteur: une entité aux multiples visages, in Bres J., Haillet P.P., Mellet S., Nølke H. \& Rosier L. (éds), Dialogisme et polyphonie: approches linguistiques, Bruxelles, De Boeck-Duculot, 75-94.

ANSCOMBRE J.-C. (2013), Polyphonie et représentation sémantique des marqueurs de discours. Quelques problèmes, Revue de Sémantique et Pragmatique 33-34, 7-32.

ANSCOMBRE J.-C., DONAIRE M.-L. \& HAILLET P.P. (éds) (2013), Opérateurs discursifs du français. Éléments de description sémantique et pragmatique, Berne, Peter Lang.

DONAIRE M.-L. (2006), Les dialogues intérieurs à la langue, Le Français Moderne 74: 1, 58-70.

DUCROT O. (1980), Analyses pragmatiques, Communication 32, 11-60.

HERMOSO A. (à paraître), Confidentiellement et entre nous: de la manière de dire à l'attitude de monstration, in Haillet P.P., Petit G. \& Salvador X.-L. (éds), Dénominations, lexique et discours, Paris, Éditions Honoré Champion.

MARQUE-PUCHEU C. (2010), Entre nous marqueur dialogique interlocutif et intralocutif dissymétrique, Colloque international Dialogisme: langue, discours, septembre 2010, Montpellier. http://recherche.univ-montp3.fr/praxiling/spip.php?article264, $08 / 08 / 2012$.

MOLINIER C. (2009), Les adverbes d'énonciation: comment les définir et les sous-classifier, Langue Française 161, 9-21.

NØLKE H. (1989), Modality and polyphony. A study of some French adverbials, Travaux du Cercle Linguistique de Copenhague 23, 45-63.

NØLKE H. (1990), Les adverbiaux contextuels: problèmes de classification, Langue Française 88, 12-27.

POP L. (2001), Adverbes de texte, L'information grammaticale 91, 13-19.

REY-DEBOVE J. \& REY A. (2002), Le Petit Robert, Paris, Dictionnaires Le Robert. 